\title{
Update in Chronic Obstructive Pulmonary Disease 2017
}

\author{
William Z. Zhang ${ }^{1,2}$, Kazunori Gomi ${ }^{1}$, Seyed Babak Mahjour ${ }^{1}$, Fernando J. Martinez ${ }^{1}$, and Renat Shaykhiev ${ }^{1}$ \\ ${ }^{1}$ Department of Medicine, Weill Cornell Medical College, New York, New York; and ${ }^{2}$ New York Presbyterian Hospital, Weill Cornell \\ Medical Center, New York, New York
}

Chronic obstructive pulmonary disease (COPD) remains an area of considerable interest among healthcare providers, clinical investigators, and lung biologists, not in small part because of its profound impact on health care, both at present and forthcoming (1). According to the Global Burden of Disease Study, more than 250 million people suffer from COPD worldwide, and about 3 million people died from it in 2016 $(2,3)$, which places COPD in the top four in causes of death (4). In this Update, we summarize the progress made in 2017 in understanding the pathogenesis and clinical aspects of COPD and discuss how this knowledge can lead to novel therapies.

\section{Origins and Natural History of COPD}

In 2017, the iconic article by Fletcher and Peto, in which they pioneered the concept of the natural history of COPD as a gradual accelerated decline in $\mathrm{FEV}_{1}$ due to smoking, celebrated its 40th anniversary with an excellent tribute by Vestbo and Lange (5). In this tribute, the authors discussed recent progress in understanding the developmental trajectories of COPD, beyond the simplistic perception of excessive $\mathrm{FEV}_{1}$ decline as an obligatory or exclusive pathway to COPD. For example, the inability to attain maximal lung function in early adulthood, a starting point for physiological, age-related decline in $\mathrm{FEV}_{1}$, may lead to COPD in susceptible individuals without excessive $\mathrm{FEV}_{1}$ decline after that point.
In support of this concept, Bui and colleagues (6) analyzed data from the Tasmanian Longitudinal Health Study, in which 1,389 children born in 1961 were evaluated spirometrically at age 7 years and then at age 45 years. They found that lower $\mathrm{FEV}_{1} / \mathrm{FVC}$ ratio at age 7 years is associated with an increased risk of COPD and asthma-COPD overlap syndrome, but not asthma alone. Among subjects with COPD, a higher proportion of never-smokers than ever-smokers had lower $\mathrm{FEV}_{1} / \mathrm{FVC}$ at age 7 years. This observation implies that early-life factors may interfere with lung maturation during childhood and contribute to COPD development independent from smoking.

Different insights were provided in the U.K. study by Allinson and colleagues (7), who analyzed data for 5,362 individuals born in March 1946 whose lung function was assessed between ages 43 and 60 to 64 years. In this study, individuals with adverse earlylife exposures, such as infant respiratory infection and home overcrowding, developed lower $\mathrm{FEV}_{1}$ and $\mathrm{FVC}$ values by age 43 years, but only if they had also become smokers. This suggests the possibility that smoking may lead to COPD by preventing the restoration of lung maturation after negative early-life exposures.

\section{Genetic Determinants}

Genetic susceptibility is one of the determinants of lung function and risk for COPD. The International COPD Genetics
Consortium study (8) analyzed 63,192 individuals to identify candidate loci, which were then tested using the U.K. BiLEVE (Biobank Lung Exome Variant Evaluation) cohort. In addition to capturing previously known COPD risk genes, this study identified several genetic variants associated with disease and/or lung function involving DSP (desmoplakin), coding for a desmosome component, and SFTPD (surfactant protein D). A parallel study included 48,943 subjects from the U.K. BiLEVE cohort (9) and identified genes associated with $\mathrm{FEV}_{1}$ and FVC, including CHRM3 (cholinergic receptor muscarinic 3 ) and genes related to the phosphoinositide 3-kinase pathway.

Boueiz and colleagues (10) evaluated association of genetic variations with computed tomography (CT) data in 11,532 subjects and found that specific loci near known COPD risk genes HHIP (hedgehoginteracting protein) and CHRNA5 (cholinergic receptor nicotinic $\alpha 5$ subunit) have the strongest association with emphysema; they identified new associations, including those related to transforming growth factor- $\beta, N F-\kappa B$ (nuclear factor-кB), and endothelial cell signaling. By relating their identified SNP data to the Encyclopedia of DNA Elements (ENCODE) and Roadmap Epigenomics project databases, they found that COPDassociated loci are frequently detected within annotated enhancer regions that regulate gene expression in fetal lung, fibroblasts, and lymphoid cells.

Because of psychosocial and biological factors, COPD is known to affect men and

(Received in original form January 16, 2018; accepted in final form April 24, 2018)

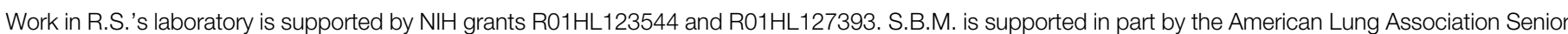
Research Training Fellowship and Weill Cornell Clinical and Translational Science Center TL1 Training Award.

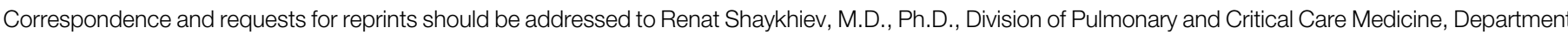
of Medicine, Weill Cornell Medical College, 1300 York Avenue, A-324, New York, NY. E-mail: res2003@med.cornell.edu.

Am J Respir Crit Care Med Vol 197, Iss 12, pp 1534-1539, Jun 15, 2018

Copyright (C) 2018 by the American Thoracic Society

Originally Published in Press as DOI: 10.1164/rccm.201801-0113UP on April 24, 2018

Internet address: www.atsjournals.org 
women differently. Amaral and colleagues (11) evaluated the relationship between sex, smoking patterns, and lung function in the U.K. Biobank cohort and found that when adjusted to the same dose of smoking, women are at a greater risk for airflow obstruction than men, suggesting that females may respond differently to smoking. In their search for sex-specific genetic traits that underlie the differential susceptibility of women to COPD development, Hardin and colleagues (12) identified a SNP in CELSR1 (cadherin EGF LAG seven-pass G-type receptor 1), a cadherin-related gene implicated in lung development, as being associated with COPD in females but not males. CELSR1 expression was higher in female versus male fetal lung during the lung development period when airway morphogenesis occurs. SNPs in the CELSR1 gene were not associated with COPD in never-smokers, suggesting that they may influence response to smoking.

\section{Pre-COPD/Early COPD}

Although COPD is defined using a postbronchodilator $\mathrm{FEV}_{1} / \mathrm{FVC}$ ratio less than 0.70 on spirometry, this cutoff is arbitrary, and there is accumulating evidence pointing toward the existence of a prediagnostic stage ("pre-COPD" or "early COPD”) that is characterized by COPDlike symptoms and manifestations of airway remodeling and emphysema typical for COPD (13). Numerous imaging-based methodologies have been established to estimate the presence and extent of structural abnormalities in the lung in smokers not meeting criteria for obstruction on spirometry and to monitor the progression of these abnormalities over time. One of these metrics, the ratio of expiratory to inspiratory mean lung density has been developed by Bodduluri and colleagues (14) as an alternative measure of gas trapping that adjusts for emphysema and has stronger associations with small airways disease than existing measures of gas trapping. By measuring the expiratory to inspiratory mean lung density ratio in more than 8,000 participants of the COPDGene study, they found that in more than $26 \%$ of smokers without COPD, lung regions considered normal on CT scan using standard thresholds show signs of gas trapping, and this was independently associated with $\mathrm{FEV}_{1}$ and progressively increased from mild to severe COPD (14). These changes may eventually progress into spirometrically detectable airflow obstruction and provide an explanation for the respiratory symptoms in "healthy" smokers. Another marker of early COPD development could be the overproduction of mucus, indicative of chronic bronchitis known to precede COPD in a considerable subset of smokers (15). By analyzing 917 samples from subjects who participated in SPIROMICS (Subpopulations and Intermediate Outcome Measures in COPD Study), Kesimer and colleagues (16) found increased concentrations of airway mucins in the sputum of smokers without COPD compared with never-smokers. In subjects with COPD, mucin concentration correlated with disease severity and a history of exacerbations.

\section{Undiagnosed COPD and Diagnostic Instability}

Although some symptomatic smokers do not meet spirometric criteria for COPD, many patients remain undiagnosed despite having airflow obstruction. Çolak and colleagues (17) analyzed data from 95,288 individuals from the Copenhagen General Population Study and found that of the subjects who met criteria for COPD, $78 \%$ were undiagnosed, and $71 \%$ of these undiagnosed patients with COPD were symptomatic and had elevated risks for exacerbations, death, and other adverse outcomes (17).

Despite the fact that research efforts to extend the use of spirometry to diverse populations (18) and to stratify disease cohorts (19) have been fruitful, in many ways spirometry remains problematic even as a basic assessment tool. By analyzing two large COPD cohorts, the Lung Health Study and the CanCOLD (Canadian Cohort of Obstructive Lung Disease) study, Aaron and colleagues (20) found that 20\% of patients with mild to moderate COPD had their $\mathrm{FEV}_{1} / \mathrm{FVC}$ cross into the nondiagnostic range of greater than 0.70 over a 5-year follow-up period. This phenomenon, termed "diagnostic instability," was especially true among patients whose initial $\mathrm{FEV}_{1} / \mathrm{FVC}$ was close to 0.70 . "Diagnostic reversal" was also observed in this analysis, where up to $10 \%$ of patients had their $\mathrm{FEV}_{1} / \mathrm{FVC}$ improve and stabilize above the 0.70 threshold. Encouragingly, sustained smoking cessation resulted in an odds ratio of 2.92 for having diagnostic reversal compared with ongoing smokers (20), highlighting the importance of smoking cessation in changing the natural history of COPD, especially in patients with mild disease.

\section{Structural Changes in the Lung}

Although $\mathrm{FEV}_{1}$ decline is influenced by both airway remodeling and reduced lung elastic recoil due to emphysema, recent evidence suggests that it is the former-the derangement of small airways-that ultimately results in airflow limitation in COPD (21). Characteristic features of such derangement are the disappearance of small airways and the remodeling of the remaining bronchioles, which may precede emphysema (21). To provide novel insights into the relationship between airway remodeling and emphysema, Tanabe and colleagues (22) used micro-CT technology that enables visualization of small airways to evaluate preterminal bronchioles in patients with centrilobular and panlobular emphysema (CLE and PLE, respectively) compared with normal lungs. This study showed that, although the alveolar region is considered the primary site of emphysema, preterminal bronchioles undergo structural changes in CLE and PLE lungs: they become shorter and lose their circular luminal shape. Compared with normal and PLE lungs, marked changes in the wall of preterminal bronchioles were observed in CLE, which correlated with fewer alveolar attachments, further supporting the concept that the pathogenesis of emphysema in COPD might begin with the derangement of small airways $(21,22)$.

Once established, emphysema may propagate itself by spreading to surrounding normal tissue. Changes in transpulmonary pressure due to breathing or coughing may cause over time sequential destruction of alveolar walls surrounding emphysematous areas, where the mechanical stability of extracellular matrix is compromised because of ongoing disease $(23,24)$. To evaluate this possibility, Bhatt and colleagues (23) analyzed volumetric CT scans obtained from 714 subjects enrolled in the COPDGene study. By quantifying regional parenchymal volume change with 
respiration in the emphysematous and surrounding "normal" areas of the lung, the authors found that in individuals with emphysema, normal-appearing lung regions on $\mathrm{CT}$ have altered regional expansion, and these areas of mechanically affected lung (MAL) have a spatial relationship with emphysematous areas. The number of MAL areas could independently predict lung function change over time, suggesting that MAL regions could represent areas of "lung at risk" for emphysema progression (23).

\section{Biologic Mechanisms}

The destructive character of pathologic changes in COPD suggests that failure of physiologic regenerative mechanisms to maintain tissue integrity may represent the unifying principle of disease pathogenesis. Reduced regenerative capacity of airway epithelial progenitors could contribute to the loss of airways observed in COPD, whereas the pathogenesis of airway remodeling may involve pathologic reprogramming of these progenitor cells by aberrantly activated signaling pathways. Zuo and colleagues (25) identified a novel mechanism of interconnected pathogenesis of all major airway epithelial lesions in COPD, which involves a step-wise activation of the epidermal growth factor (EGF) receptor in airway basal progenitors. As a triggering event, smoking-induced EGF shifts basal progenitors toward squamous differentiation accompanied by production of another EGF receptor ligand, amphiregulin, which promotes basal cell and mucous cell hyperplasia. By comparing the transcriptomes of proximal and distal airway epithelium, Yang and colleagues (26) identified region-specific signatures and found that the distal airway epithelium in COPD undergoes smoking-dependent transition toward a proximal airwaylike pattern. This repatterning was mediated by EGF signaling in small airway basal progenitors and occurred independently from classic histologic lesions (26), implying that it may represent an early event during disease development (27).

Altered epithelial differentiation may contribute to decreased airway host defense potential. By analyzing 1,104 small airways from donors with and without COPD, Polosukhin and colleagues (28) observed a deficiency of secretory IgA (sIgA) on the surface of small airways from subjects with COPD, which was associated with mucous and squamous metaplasia. sIgA is delivered to the apical surface via the polymeric immunoglobulin receptor, whose expression is dependent on epithelial differentiation. COPD airways with low sIgA levels showed higher levels of bacterial DNA, thicker walls, and increased number of neutrophils and macrophages (28), linking altered airway epithelial differentiation, host defense, tissue remodeling, and inflammation. As further evidence supporting this link, Kedzierski and colleagues found that airway epithelial cells from subjects with COPD fail to upregulate SOCS (suppressor of cytokine signaling)-5 in response to influenza virus infection, resulting in increased EGF receptor signaling that facilitates viral pathogenesis (29) and also mediates airway epithelial remodeling (25).

Altered maintenance of the alveolar epithelium by region-specific progenitors, type 2 alveolar cells, may contribute to pathogenesis of emphysema. Baarsma and colleagues (30) found that alveolar epithelial repair mediated by canonical Wnt (wingless-type MMTV integration site family) signaling in type 2 cells can be inhibited in COPD because of upregulation of a noncanonical signaling mediated by Wnt5A induced in lung fibroblasts undergoing smoking-associated senescence. As another mechanism for decreased canonical Wnt signaling relevant to the pathogenesis of emphysema, SkronskaWasek and colleagues (31) showed reduced expression of Wnt receptor Frizzled-4 in the lungs of subjects with COPD that correlated with smoking history and severity of airflow obstruction. Inflammation may also play a role in mediating destructive changes in the lung parenchyma. Increased numbers of macrophages and $\mathrm{T}$ cells, accompanied by upregulation of a molecular signature of T-helper cell type 1-related inflammation, have been found in the small airway and alveolar tissue in association with emphysema (32).

\section{Exacerbations}

COPD is not only a disease of steady decline in lung function but also one that is punctuated by difficult-to-predict exacerbations with significant clinical consequences. Han and colleagues (33) analyzed the pattern of exacerbations among patients with 3 years of complete follow-up data in the SPIROMICS cohort. Although increased exacerbation rate correlated strongly with a history of exacerbations, the overall pattern was highly heterogeneous, with only $2 \%$ of patients having two or more exacerbations in each of the 3 years that would warrant classification as "frequent exacerbator." There are clearly subgroups of patients with COPD who are at higher risk for having exacerbations, but "frequent exacerbator" appears to be a heterogeneous phenotype that is stable neither in a population nor across time. By the same token, Dransfield and colleagues (34) examined the follow-up data on 2,000 patients from the COPDGene study and observed that the excessive decrease in $\mathrm{FEV}_{1}$ after a severe exacerbation was the greatest in patients with Global Initiative for Chronic Obstructive Lung Disease (GOLD) stage 1 COPD, an extra $87 \mathrm{ml} / \mathrm{yr}$. Although the mean rate of exacerbations was higher in patients with GOLD 4 than GOLD 1 COPD, more than $20 \%$ of patients with GOLD 1 COPD experienced at least one exacerbation during the follow-up, with $8 \%$ experiencing a severe exacerbation. With the addition of a post hoc analysis of another large clinical trial showing the efficacy of the combination of inhaled corticosteroid and long-acting $\beta$-agonist in reducing the rate of moderate and severe exacerbations (35), it is more obvious than ever that preventing exacerbations should be a goal for every provider caring for patients with COPD (36).

\section{Multimorbidity}

COPD is increasingly being recognized as a multimorbid, systemic disease. For example, COPD and congestive heart failure are both diseases with an increased ventilatory drive, and Rocha and colleagues (37) showed that patients with COPD-congestive heart failure overlap syndrome, particularly those with resting hypocapnia, have worse ventilatory efficiency and more dynamic hyperinflation, likely resulting in more severe dyspnea and worse exertional tolerance. The proposed mechanisms of left ventricular dysfunction associated with COPD include mechanical effects from hyperinflation as well as pulmonary 
microvascular damage induced by smoking, an endothelial injury phenotype recently reported also in the kidneys of patients who smoke (38). These findings suggest that medications traditionally used in cardiovascular disease may have a role in the management of COPD, altering pathways not classically believed to be pertinent in COPD pathogenesis.

\section{Treatment}

\section{Smoking Cessation}

Many patients continue to smoke after being diagnosed with COPD, even though smoking cessation is the only etiology-oriented treatment approach that has been shown to prevent disease progression. This was again demonstrated in a recent U.K. study, in which Josephs and colleagues (39) analyzed the data of 16,479 patients with COPD having different smoking histories and found that compared with active smokers, ex-smokers had reduced risk of death, hospitalization, and emergency department attendance, regardless of age or lung function.

\section{Pharmacologic Therapy}

Whether patients with mild COPD warrant pharmacologic therapy is currently unknown. To address this question, Zhou and colleagues (40) conducted a clinical trial in which 841 patients with mild to moderate COPD were randomized to tiotropium versus placebo. They showed a 157-ml between-group difference in prebronchodilator $\mathrm{FEV}_{1}$ at 24 months, as well as slower decline in $\mathrm{FEV}_{1}$ and longer time to first exacerbation in the tiotropium group. Although the high inhaler compliance rates seen in this study cannot be expected among minimally symptomatic patients outside of a trial setting, this study raises interesting questions about the treatment of mild COPD.

Regarding the management of moderate to severe COPD, the FULFILL (Lung Function and Quality of Life Assessment in Chronic Obstructive Pulmonary Disease with Closed Triple Therapy) trial (41) demonstrated that triple therapy result in improved $\mathrm{FEV}_{1}$ and respiratory symptoms, as well as a reduction in the rate of moderate/severe exacerbations, compared with inhaled corticosteroid/long-acting $\beta$-agonist. In the TRINITY (Single Inhaler Extrafine Triple Therapy versus Long-Acting Muscarinic
Antagonist Therapy for Chronic Obstructive Pulmonary Disease) trial, 2,691 patients with severe COPD were randomized to a single-inhaler triple therapy, triple therapy in separate inhalers, or long-acting muscarinic agonist alone (42). Fixed-combination therapy was superior to tiotropium monotherapy in reducing the yearly rate of moderate to severe exacerbations by about $20 \%$, the first long-term study to show this effect when comparing triple therapy to long-acting muscarinic agonist alone. Fixedcombination single-inhaler triple therapy was neither inferior nor superior to opencombination triple therapy in this study, but future trials should consider reexamining whether a single-inhaler triple therapy would be superior to multiinhaler therapy in real-world patients.

\section{Novel Therapies}

One approach to treating COPD could be the use of therapies that target pathways important for COPD pathogenesis that have already been found to be effective for other diseases. An example of this is using monoclonal antibodies against IL-5, a cytokine that activates various aspects of eosinophil biology, in patients whose disease is driven by eosinophilic inflammation, as is known for asthma and probably is relevant for some patients with COPD (43). Clinical trials already frequently analyze outcomes for interactions with eosinophils post hoc (44), with earlier observations suggesting that increased blood eosinophils correlate with favorable response to glucocorticoid therapy; more recently, in the SPIROMICS study, Hastie and colleagues (45) found that high concentrations of sputum eosinophils define a subset of subjects with COPD with more severe airflow obstruction and more frequent exacerbations. To address whether targeting IL-5 will provide therapeutic benefit in COPD, Pavord and colleagues (46) randomized patients with moderate to severe COPD with elevated blood eosinophil counts to receive the monoclonal antibody mepolizumab against standard care. Although the results of this study were somewhat disappointing, it appears that anti-IL-5 therapy is effective in a subset of patients with COPD, and further studies are needed to elaborate on this.

\section{Nonpharmacological Approaches}

Treatment modalities outside of pharmacologic therapy continue to be explored for patients with severe COPD, especially in means of lung volume reduction (LVR). Kemp and colleagues (47) conducted the first multicenter randomized controlled trial using endobronchial valves for LVR in patients with COPD on optimal medical management without collateral ventilation in the target lobe. At 3 months and 6 months after the procedure, patients in the endobronchial valves group had improved $\mathrm{FEV}_{1}$, 6-minute-walk distance, and respiratory symptoms. However, adverse events continue to be a concern, as almost $30 \%$ of patients who received an endobronchial valve developed a pneumothorax. It will be of interest to see if risk stratification scores currently applied in assessment for surgical LVR (48) can also improve patient selection for endoscopic LVR procedures.

In the management of hospitalized patients with COPD exacerbations, a recent multicenter clinical trial evaluated the role of helium-oxygen mixture (Heliox) in the prevention of endotracheal intubation in patients with hypercapnic COPD exacerbations requiring noninvasive ventilation (NIV) (49). Because of the low overall rate of the primary outcome, the study was stopped prematurely after 445 patients, at which point there was no significant difference between the groups. Another study showed that with stringent patient selection, aggressive NIV titration, and adherence monitoring, discharging patients with severe COPD with home NIV may prevent further exacerbations or readmissions to the hospital in this group of high-risk patients (50).

\section{Pulmonary Rehabilitation}

Nolan and colleagues (51) sought to improve on the approach to pulmonary rehabilitation by using pedometer-directed step targets to improve patients' exercise tolerance but found no difference between the study and control groups in the amount of time expending at least 3 metabolic equivalents after 8 weeks of intervention. Although there is likely a role for the integration of new technology in improving the physical activity level of patients with COPD (52), this study shows that this is not accomplished by providing numerical goals alone (53).

\section{Future Perspectives}

Despite the magnitude of the problem and significant progress in understanding the 
pathophysiology and clinical aspects of COPD, specific mechanisms underlying COPD development and heterogeneity remain poorly understood, and no therapy so far has been shown to prevent long-term decline in lung function (54). Although standard COPD therapies target common pathways mediating symptoms in many patients, distinct mechanisms may underlie unique "natural histories" and clinical phenotypes in individual patients or groups of patients. Future therapies would ultimately require a transition from classic, population-based thinking about disease toward precision medicine approaches on the basis of the knowledge of specific pathophysiological patterns and diseasedriving mechanisms in each patient. This could be achieved through a broader use of patient-derived organotypic models of
COPD, such as airway and lung organoids (55-57), in which disease mechanisms can be identified, reconstructed, and targeted in a tissue- and patient-specific fashion.

Author disclosures are available with the text of this article at www.atsjournals.org.

Acknowledgment: The authors thank Dr. David Price and Dr. Suzanne Cloonan for their useful suggestions in reviewing the manuscript.

\section{References}

1. Khakban A, Sin DD, FitzGerald JM, McManus BM, Ng R, Hollander Z, et al. The projected epidemic of chronic obstructive pulmonary disease hospitalizations over the next 15 years: a population-based perspective. Am J Respir Crit Care Med 2017;195:287-291.

2. Abajobir AA, Abate KH, Abbafati C, Abbas KM, Abd-Allah F, Abdulkader RS, et al.; GBD 2016 Disease and Injury Incidence and Prevalence Collaborators. Global, regional, and national incidence, prevalence, and years lived with disability for 328 diseases and injuries for 195 countries, 1990-2016: a systematic analysis for the Global Burden of Disease Study 2016. Lancet 2017;390:1211-1259.

3. Abajobir AA, Abbafati C, Abbas KM, Abd-Allah F, Abera SF, Aboyans V, et al.; GBD 2016 Causes of Death Collaborators. Global, regional, and national age-sex specific mortality for 264 causes of death, 19802016: a systematic analysis for the Global Burden of Disease Study 2016. Lancet 2017;390:1151-1210.

4. Vogelmeier CF, Criner GJ, Martinez FJ, Anzueto A, Barnes PJ, Bourbeau J, et al. Global Strategy for the Diagnosis, Management, and Prevention of Chronic Obstructive Lung Disease 2017 report: GOLD executive summary. Am J Respir Crit Care Med 2017;195:557-582.

5. Vestbo J, Lange P. Fletcher and Peto 40 years on: a tribute and reflection. Am J Respir Crit Care Med 2017;195:1420-1422.

6. Bui DS, Burgess JA, Lowe AJ, Perret JL, Lodge CJ, Bui M, et al. Childhood lung function predicts adult chronic obstructive pulmonary disease and asthma-chronic obstructive pulmonary disease overlap syndrome. Am J Respir Crit Care Med 2017;196:39-46.

7. Allinson JP, Hardy R, Donaldson GC, Shaheen SO, Kuh D, Wedzicha JA. Combined impact of smoking and early-life exposures on adult lung function trajectories. Am J Respir Crit Care Med 2017;196:1021-1030.

8. Hobbs BD, de Jong K, Lamontagne M, Bossé Y, Shrine N, Artigas MS, et al.; COPDGene Investigators; ECLIPSE Investigators; LifeLines Investigators; SPIROMICS Research Group; International COPD Genetics Network Investigators; UK BiLEVE Investigators; International COPD Genetics Consortium. Genetic loci associated with chronic obstructive pulmonary disease overlap with loci for lung function and pulmonary fibrosis. Nat Genet 2017;49:426-432.

9. Wain LV, Shrine N, Artigas MS, Erzurumluoglu AM, Noyvert B, BossiniCastillo L, et al.; Understanding Society Scientific Group; GeisingerRegeneron DiscovEHR Collaboration. Genome-wide association analyses for lung function and chronic obstructive pulmonary disease identify new loci and potential druggable targets. Nat Genet 2017;49:416-425.

10. Boueiz A, Lutz SM, Cho MH, Hersh CP, Bowler RP, Washko GR, et al.; COPDGene and ECLIPSE Investigators. Genome-wide association study of the genetic determinants of emphysema distribution. Am J Respir Crit Care Med 2017;195:757-771.

11. Amaral AFS, Strachan DP, Burney PGJ, Jarvis DL. Female smokers are at greater risk of airflow obstruction than male smokers UK Biobank. Am J Respir Crit Care Med 2017;195:1226-1235.

12. Hardin M, Cho MH, Sharma S, Glass K, Castaldi PJ, McDonald ML, et al.; COPDGene and Evaluation of COPD Longitudinally to Identify Predictive Surrogate End-Points Investigators. Sex-based genetic association study identifies CELSR1 as a possible chronic obstructive pulmonary disease risk locus among women. $A m \mathrm{~J}$ Respir Cell Mol Biol 2017;56:332-341.
13. Rodriguez-Roisin R, Han MK, Vestbo J, Wedzicha JA, Woodruff PG, Martinez FJ. Chronic respiratory symptoms with normal spirometry: a reliable clinical entity? Am J Respir Crit Care Med 2017;195: 17-22.

14. Bodduluri S, Reinhardt JM, Hoffman EA, Newell JD Jr, Nath H, Dransfield MT, et al.; COPDGene Investigators. Signs of gas trapping in normal lung density regions in smokers. Am J Respir Crit Care Med 2017;196:1404-1410.

15. Wedzicha JA. Airway mucins in chronic obstructive pulmonary disease. N Engl J Med 2017;377:986-987.

16. Kesimer M, Ford AA, Ceppe A, Radicioni G, Cao R, Davis CW, et al. Airway mucin concentration as a marker of chronic bronchitis. N Engl J Med 2017;377:911-922.

17. Çolak Y, Afzal S, Nordestgaard BG, Vestbo J, Lange P. Prognosis of asymptomatic and symptomatic, undiagnosed COPD in the general population in Denmark: a prospective cohort study. Lancet Respir Med 2017;5:426-434.

18. LaVange L, Davis SM, Hankinson J, Enright P, Wilson R, Barr RG, et al. Spirometry reference equations from the HCHS/SOL (Hispanic Community Health Study/Study of Latinos). Am J Respir Crit Care Med 2017;196:993-1003.

19. Tejero E, Prats E, Casitas R, Galera R, Pardo P, Gavilán A, et al. Classification of airflow limitation based on z-score underestimates mortality in patients with chronic obstructive pulmonary disease. Am J Respir Crit Care Med 2017;196:298-305.

20. Aaron SD, Tan WC, Bourbeau J, Sin DD, Loves RH, MacNeil J, et al.; Canadian Respiratory Research Network. Diagnostic instability and reversals of chronic obstructive pulmonary disease diagnosis in individuals with mild to moderate airflow obstruction. Am J Respir Crit Care Med 2017;196:306-314.

21. Hogg JC, Paré PD, Hackett T-L. The contribution of small airway obstruction to the pathogenesis of chronic obstructive pulmonary disease. Physiol Rev 2017;97:529-552.

22. Tanabe N, Vasilescu DM, McDonough JE, Kinose D, Suzuki M, Cooper JD, et al. Micro-computed tomography comparison of preterminal bronchioles in centrilobular and panlobular emphysema. Am J Respir Crit Care Med 2017;195:630-638.

23. Bhatt SP, Bodduluri S, Hoffman EA, Newell JD Jr, Sieren JC, Dransfield MT, et al.; COPDGene Investigators. Computed tomography measure of lung at risk and lung function decline in chronic obstructive pulmonary disease. Am J Respir Crit Care Med 2017;196:569-576.

24. Parr DG. Quantifying the lung at risk in chronic obstructive pulmonary disease: does emphysema beget emphysema? Am J Respir Crit Care Med 2017;196:535-536.

25. Zuo WL, Yang J, Gomi K, Chao I, Crystal RG, Shaykhiev R. EGFamphiregulin interplay in airway stem/progenitor cells links the pathogenesis of smoking-induced lesions in the human airway epithelium. Stem Cells 2017;35:824-837.

26. Yang J, Zuo W-L, Fukui T, Chao I, Gomi K, Lee B, et al. Smokingdependent distal-to-proximal repatterning of the adult human smal airway epithelium. Am J Respir Crit Care Med 2017;196:340-352.

27. Bartel S, Bhakta N, Christenson S. More than meets the eye: cigarette smoke induces genomic changes in the small airway epithelium independent of histologic changes. Am J Respir Crit Care Med 2017; 196:260-262. 
28. Polosukhin VV, Richmond BW, Du RH, Cates JM, Wu P, Nian H, et al. Secretory IgA deficiency in individual small airways is associated with persistent inflammation and remodeling. Am J Respir Crit Care Med 2017;195:1010-1021.

29. Kedzierski L, Tate MD, Hsu AC, Kolesnik TB, Linossi EM, Dagley L, et al. Suppressor of cytokine signaling (SOCS) 5 ameliorates influenza infection via inhibition of EGFR signaling. eLife 2017;6:1-27.

30. Baarsma HA, Skronska-Wasek W, Mutze K, Ciolek F, Wagner DE, John-Schuster G, et al. Noncanonical WNT-5A signaling impairs endogenous lung repair in COPD. J Exp Med 2017;214:143-163.

31. Skronska-Wasek W, Mutze K, Baarsma HA, Bracke KR, Alsafadi HN, Lehmann $\mathrm{M}$, et al. Reduced frizzled receptor 4 expression prevents WNT/ $\beta$-catenin-driven alveolar lung repair in chronic obstructive pulmonary disease. Am J Respir Crit Care Med 2017;196:172-185.

32. Suzuki M, Sze MA, Campbell JD, Brothers JF II, Lenburg ME, McDonough JE, et al. The cellular and molecular determinants of emphysematous destruction in COPD. Sci Rep 2017;7:9562.

33. Han MK, Quibrera PM, Carretta EE, Barr RG, Bleecker ER, Bowler RP, et al.; SPIROMICS investigators. Frequency of exacerbations in patients with chronic obstructive pulmonary disease: an analysis of the SPIROMICS cohort. Lancet Respir Med 2017;5:619-626.

34. Dransfield MT, Kunisaki KM, Strand MJ, Anzueto A, Bhatt SP, Bowler RP, et al.; COPDGene Investigators. Acute exacerbations and lung function loss in smokers with and without chronic obstructive pulmonary disease. Am J Respir Crit Care Med 2017;195:324-330.

35. Martinez FJ, Vestbo J, Anderson JA, Brook RD, Celli BR, Cowans NJ, et al.; SUMMIT Investigators. Effect of fluticasone furoate and vilanterol on exacerbations of chronic obstructive pulmonary disease in patients with moderate airflow obstruction. Am J Respir Crit Care Med 2017; 195:881-888.

36. Wedzicha JA, Calverley PMA, Albert RK, Anzueto A, Criner GJ, Hurst JR, et al. Prevention of COPD exacerbations: a European Respiratory Society/American Thoracic Society guideline. Eur Respir J 2017;50: 1602265.

37. Rocha A, Arbex FF, Sperandio PA, Souza A, Biazzim L, Mancuso F, et al. Excess ventilation in chronic obstructive pulmonary diseaseheart failure overlap: implications for dyspnea and exercise intolerance. Am J Respir Crit Care Med 2017;196:1264-1274.

38. Polverino F, Laucho-Contreras ME, Petersen H, Bijol V, Sholl LM, Choi ME, et al. A pilot study linking endothelial injury in lungs and kidneys in chronic obstructive pulmonary disease. Am J Respir Crit Care Med 2017;195:1464-1476.

39. Josephs L, Culliford D, Johnson M, Thomas M. Improved outcomes in ex-smokers with COPD: a UK primary care observational cohort study. Eur Respir J 2017;49:1602114.

40. Zhou Y, Zhong NS, Li X, Chen S, Zheng J, Zhao D, et al. Tiotropium in early-stage chronic obstructive pulmonary disease. N Engl J Med 2017;377:923-935.

41. Lipson DA, Barnacle H, Birk R, Brealey N, Locantore N, Lomas DA, et al. FULFIL trial: once-daily triple therapy for patients with chronic obstructive pulmonary disease. Am J Respir Crit Care Med 2017; 196:438-446.

42. Vestbo J, Papi A, Corradi M, Blazhko V, Montagna I, Francisco C, et al. Single inhaler extrafine triple therapy versus long-acting muscarinic antagonist therapy for chronic obstructive pulmonary disease (TRINITY): a double-blind, parallel group, randomised controlled trial. Lancet 2017;389:1919-1929.
43. McDonald CF. Eosinophil biology in COPD. N Engl J Med 2017;377: 1680-1682 10.1056/NEJMe1710326.

44. Roche N, Chapman KR, Vogelmeier CF, Herth FJF, Thach C, Fogel R, et al. Blood eosinophils and response to maintenance chronic obstructive pulmonary disease: treatment data from the FLAME Trial. Am J Respir Crit Care Med 2017;195:1189-1197.

45. Hastie AT, Martinez FJ, Curtis JL, Doerschuk CM, Hansel NN, Christenson S, et al.; SPIROMICS investigators. Association of sputum and blood eosinophil concentrations with clinical measures of COPD severity: an analysis of the SPIROMICS cohort. Lancet Respir Med 2017:5:956-967.

46. Pavord ID, Chanez P, Criner GJ, Kerstjens HAM, Korn S, Lugogo N, et al:: Mepolizumab for eosinophilic chronic obstructive pulmonary disease. N Engl J Med 2017;377:1613-1629.

47. Kemp SV, Slebos D-J, Kirk A, Kornaszewska M, Carron K, Ek L, et al.; TRANSFORM Study Team. A multicenter randomized controlled trial of zephyr endobronchial valve treatment in heterogeneous emphysema (TRANSFORM). Am J Respir Crit Care Med 2017;196: 1535-1543.

48. Greening NJ, Vaughan P, Oey I, Steiner MC, Morgan MD, Rathinam S, et al. Individualised risk in patients undergoing lung volume reduction surgery: the Glenfield BFG score. Eur Respir J 2017;49:1601766.

49. Jolliet P, Ouanes-Besbes L, Abroug F, Ben Khelil J, Besbes M, Garnero A et al.; E.C.H.O. ICU Trial Investigators. A multicenter randomized trial assessing the efficacy of helium/oxygen in severe exacerbations of chronic obstructive pulmonary disease. Am J Respir Crit Care Med 2017;195:871-880.

50. Murphy PB, Rehal S, Arbane G, Bourke S, Calverley PMA, Crook AM, et al. Effect of home noninvasive ventilation with oxygen therapy vs oxygen therapy alone on hospital readmission or death after an acute COPD exacerbation: a randomized clinical trial. JAMA 2017; 317:2177-2186

51. Nolan CM, Maddocks M, Canavan JL, Jones SE, Delogu V, Kaliaraju D, et al. Pedometer step count targets during pulmonary rehabilitation in chronic obstructive pulmonary disease: a randomized controlled trial. Am J Respir Crit Care Med 2017;195:1344-1352.

52. Demeyer H, Louvaris Z, Frei A, Rabinovich RA, de Jong C, Gimeno-Santos E, et al.; Mr Papp PROactive study group and the PROactive consortium. Physical activity is increased by a 12 -week semiautomated telecoaching programme in patients with COPD: a multicentre randomised controlled trial. Thorax 2017;72: 415-423.

53. Martinez $\mathrm{CH}$. Adding pedometers to pulmonary rehabilitation did not result in greater physical activity: an important answer, but what was the question? Am J Respir Crit Care Med 2017;195:1284-1285.

54. Rabe KF, Watz H. Chronic obstructive pulmonary disease. Lancet 2017;389:1931-1940.

55. Chen Y-W, Huang SX, de Carvalho ALRT, Ho S-H, Islam MN, Volpi S, et al. A three-dimensional model of human lung development and disease from pluripotent stem cells. Nat Cell Biol 2017;19: 542-549.

56. Tan Q, Choi KM, Sicard D, Tschumperlin DJ. Human airway organoid engineering as a step toward lung regeneration and disease modeling. Biomaterials 2017;113:118-132.

57. Barkauskas CE, Chung M-I, Fioret B, Gao X, Katsura H, Hogan BL. Lung organoids: current uses and future promise. Development 2017;144:986-997. 